\title{
Discussion of "V-Matrix Method of Solving Statistical Inference Problems" by Vlamidir Vapnik and Rauf Izmailov
}

\author{
Niharika Gauraha ${ }^{\mathrm{a}}$, Akshay Chaturvedi ${ }^{\mathrm{b}}$ \\ ${ }^{a}$ Uppsala University, Uppsala \\ ${ }^{b}$ Indian Statistical Institute, Kolkata
}

\begin{abstract}
We would like to begin by stating that we have not fully understood the formulation of V-matrix conceptually. However, We are fascinated by the idea of estimation of conditional probability function without assuming any probabilistic model. In this short discussion, we would like to present that the proposed constrained quadratic optimization problem for conditional probability estimation using v-matrix based method may not have a consistent solution always. We are sure that the paper will stimulate a deeper exploration of V-matrix based methods for inference in high-dimensional problems in future research.
\end{abstract}

\section{Direct Method of Solving Conditional Probability Function}

We mostly follow the notations in [1]. We consider estimation of the following form of conditional probability function in pattern recognition problems:

$$
p(y=1 \mid x)=\frac{p(x, y=1)}{p(x)}, \quad p(x)>0,
$$

from a training dataset of $\ell$ points

$$
\left(x_{1}, y_{1}\right), \ldots,\left(x_{\ell}, y_{\ell}\right)
$$

where $y_{i} \in\{0,1\}$ and $x_{i} \in R^{n}$. Here we assume that the training data points are IID and generated according to an unknown probability measure $p(x, y)=$ 
$p(y \mid x) p(x)$. In particular, we are looking for a conditional probability function in Reproducing Kernel Hilber Space (RKHS), in the form

$$
f(x)=\sum_{i} \alpha_{i} K\left(x_{i}, x\right)=A^{T} \mathcal{K}(x)
$$

by solving the following optimization problem

$$
\begin{aligned}
\underset{A}{\operatorname{minimize}}(Y-K A)^{T} V(Y-K A)+\gamma A^{T} K A, \\
\text { subject to } \mathbf{0} \leq A^{T} \mathcal{K}\left(x_{i}\right) \leq \mathbf{1}, \quad i=1, \ldots, \ell \\
\frac{1}{\ell} \sum_{i} A^{T} \kappa\left(x_{i}\right)=\sum_{i} y_{i} / \ell=p_{1},
\end{aligned}
$$

where $K$ is the positive semidefinite kernel matrix, $p_{1}$ is the frequency of class $y=1$, estimated from the training data, $A=\left(\alpha_{1}, \ldots \alpha_{\ell}\right)$ are unknown weights to be estimated (optimization variables). and $\mathbf{0}$ and $\mathbf{1}$ are $\ell$-dimensional vectors of zeros and ones respectively.

The above quadratic programming problem for estimation of conditional probability can be simplified as

$$
\begin{gathered}
\underset{A}{\operatorname{minimize}} A^{T}(K V K+\gamma K) A-2 A^{T} K V Y, \\
\text { subject to } K A \leq \mathbf{1}, \\
-K A \leq \mathbf{0}, \text { and } \\
\frac{1}{\ell} A^{T} K \mathbf{1}=p_{1}
\end{gathered}
$$

The kernel matrix $K$ appears as constraint matrices in the above inequality and equality constraints. Since the kernel matrices are known to be positive semi-definite, the constrains matrices for the above Quadratic Programming (QP) problem may not have full rank and there is no guarantee that the constraints will be consistent, hence no solution could be found.

\section{Illustration with XOR Example}

In this section, XOR classification problem is considered to show that the $\mathrm{V}$-matrix based conditional probability estimation algorithm (we call it $\mathrm{v}$-SVM method), fails if the constraint matrix does not have full rank. 
Consider the following four points, $X$, in two dimensional feature space and their corresponding class labels $Y$.

$$
X=\left[\begin{array}{ll}
0 & 0 \\
1 & 1 \\
0 & 1 \\
1 & 0
\end{array}\right] \quad Y=\begin{aligned}
& 0 \\
& 0 \\
& 1 \\
& 1
\end{aligned}
$$

The kernel matrix produced by INK-spline and RBF kernel function is given in the following.

$$
K_{\text {spline }}=\left[\begin{array}{llll}
0 & 0 & 0 & 0 \\
0 & 2 & 1 & 1 \\
0 & 1 & 1 & 0 \\
0 & 1 & 0 & 1
\end{array}\right] \quad K_{r b f}=\left[\begin{array}{cccc}
0 & 0.37 & 0.61 & 0.61 \\
0.27 & 1 & .61 & .61 \\
.61 & .61 & 1 & 0.37 \\
.61 & .61 & 0.37 & 1
\end{array}\right]
$$

Note that the kernel matrix produced by RBF Kernel is positive definite, however, kernel matrix produced by INK-spline of order zero is positive semidefinite. In the following we show that there is no solution exists for the XOR problem using v-SVM with INK-spline of order zero .

The Python code for fitting v-SVM model is given below.

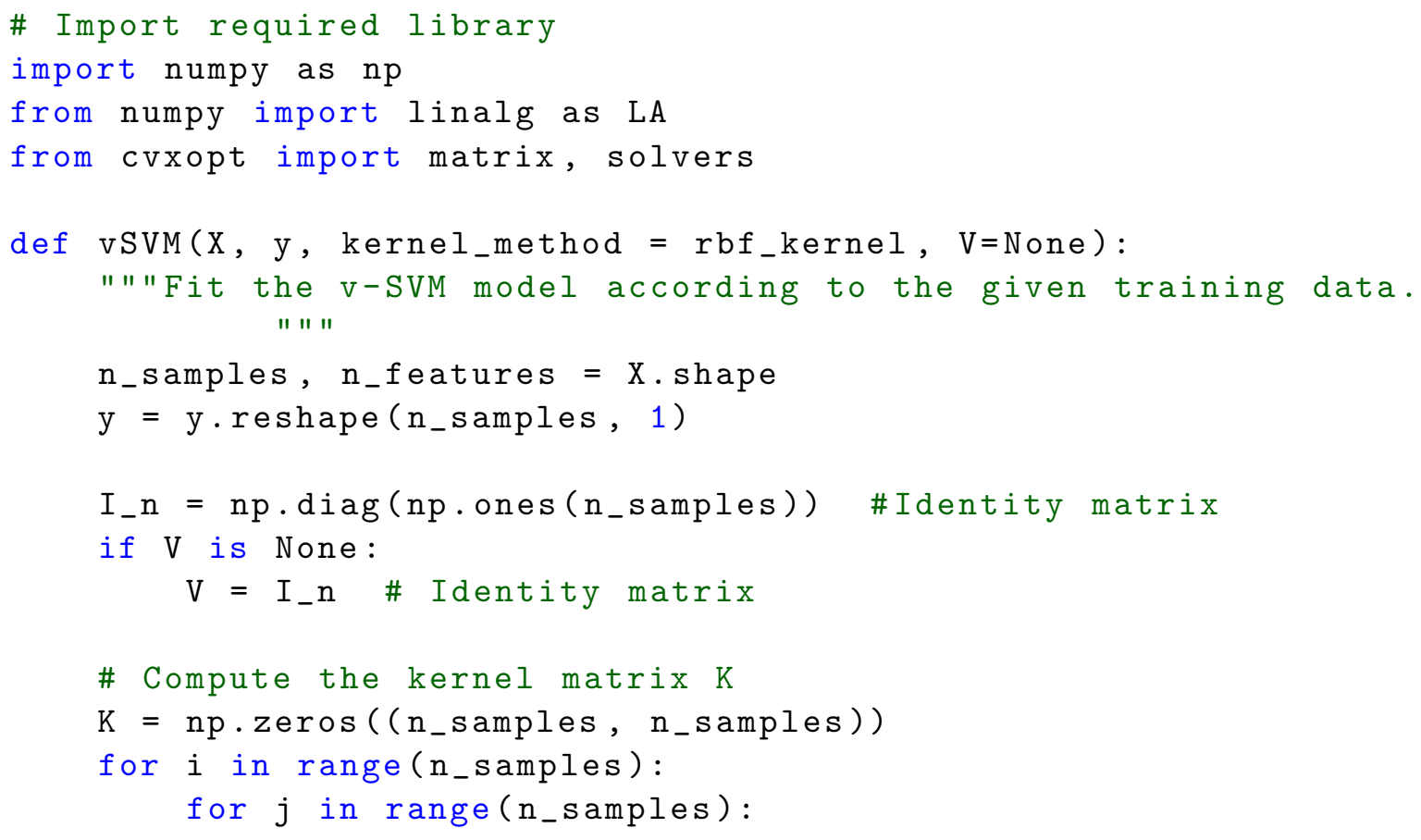




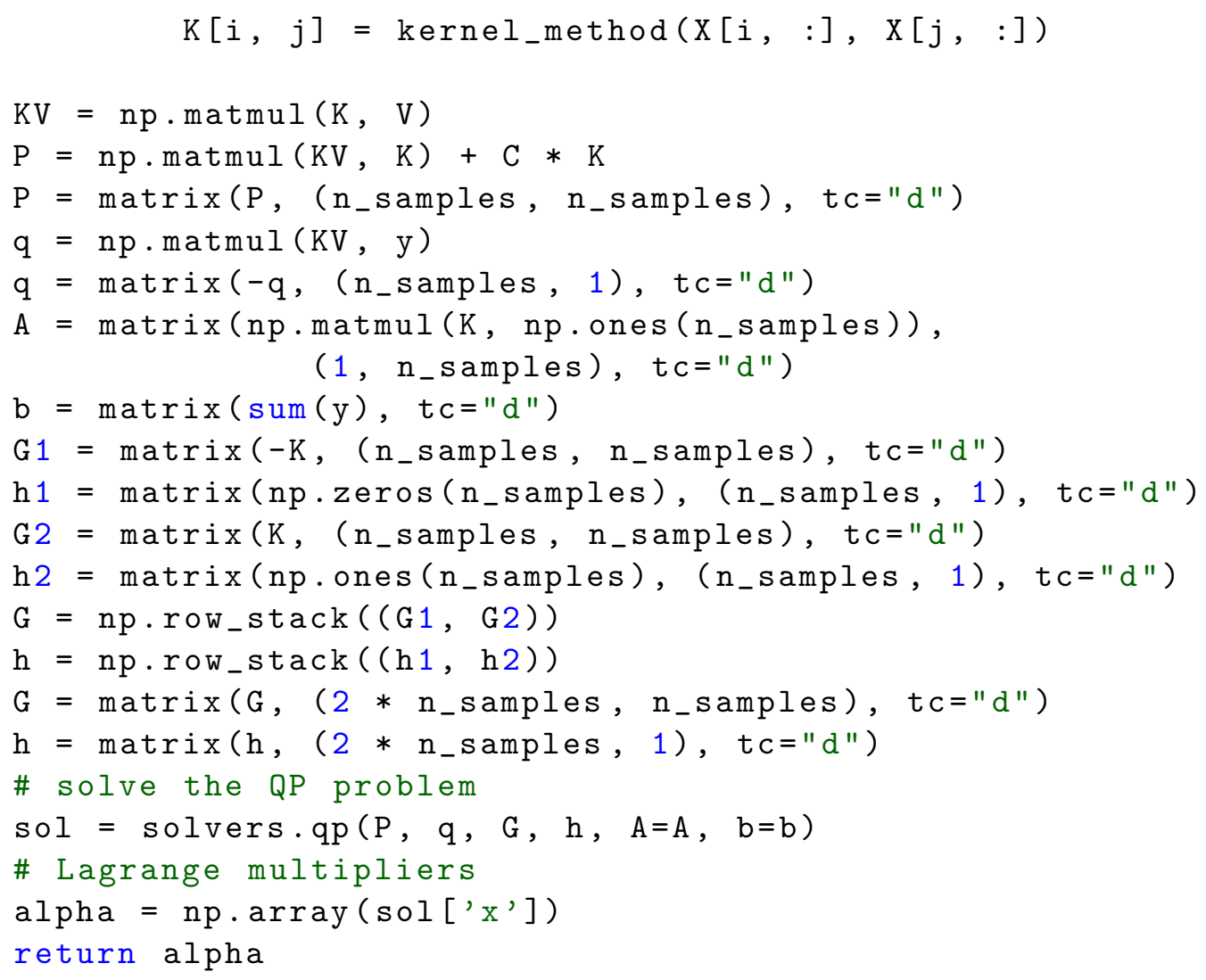

The kernel functions are defined as following.

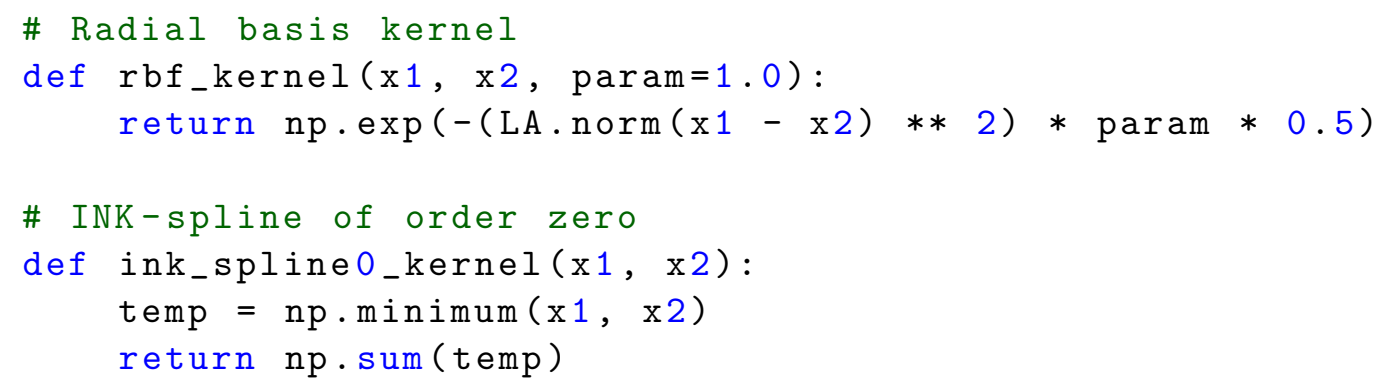

The dataset for XOR problem is defined as follows.

$\mathrm{X}=\mathrm{np} \cdot \operatorname{array}([[0,0],[1,1],[0,1],[1,0]])$

$y=n p \cdot \operatorname{array}([n p \cdot z \operatorname{zeros}(2), n p \cdot o n e s(2)])$

When we fit XOR data using v-SVM with RBF kernel, algorithms returns the optimal values.

fit1 $=\operatorname{vSVM}\left(\mathrm{X}, \mathrm{y}\right.$, kernel_method $=r b f_{-}$kernel $)$ 
However while trying to fit with the INK-spline kernel, v-SVM algorithm fails.

fit2 $=\operatorname{vSVM}(X, y$, kernel_method=ink_spline0_kernel $)$

\section{Another Example with Gaussian Mixture}

In the following we consider the Gaussian mixture model, two different Gaussian distributions for two different classes. In this case the kernel matrix produced by RBF kernel is ill-conditioned, whereas the INK-spline (with order zero) kernel function gives positive definite kernel matrix. For the farmer case the v-SVM algorithm fails, and for later case the v-SVM returns the optimal values.

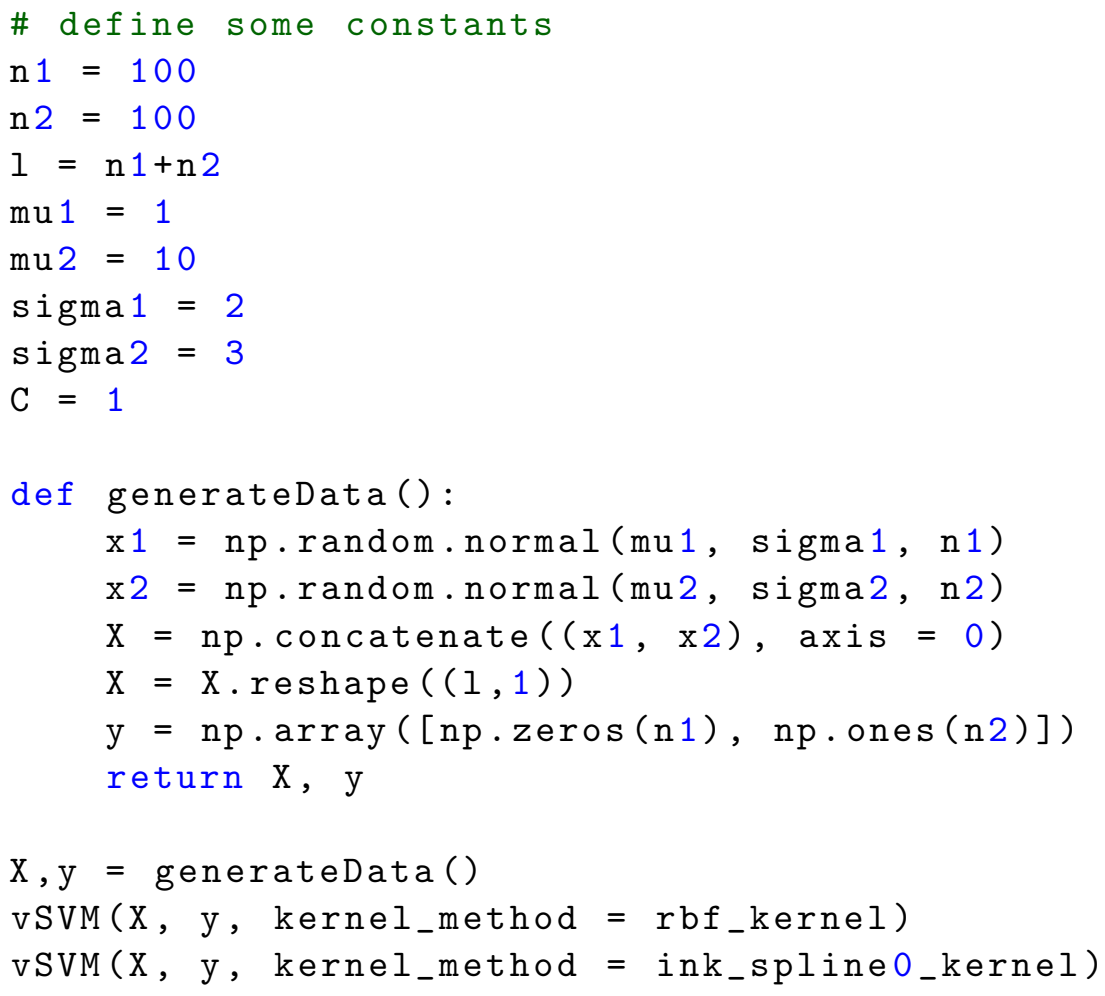

\section{Conclusion}

The V-matrix based proposed method for estimation of conditional probability function is sensitive to the kernel matrix $K$. When the kernel matrix 
is ill-conditioned the inequality constraints are inconsistent and no solution could be found. The same argument holds true for the V-matrix based proposed methods for estimation of regression function and estimation of density ratio function.

[1] V. Vapnik, R. Izmailov, V-matrix method of solving statistical inference problems., Journal of Machine Learning Research 16 (2015) (2015) 16831730 . 